\title{
Les délocalisations industrielles dans les mutations des systèmes productifs français
}

Industrial relocation and the mutations in French production systems

Dalila Messaoudi

\section{(2) OpenEdition}

\section{Journals}

Electronic version

URL: http://journals.openedition.org/rge/5153

DOI: $10.4000 /$ rge.5153

ISSN: $2108-6478$

Publisher

Association des géographes de l'Est

\section{Printed version}

Date of publication: 15 October 2014

ISSN: 0035-3213

Electronic reference

Dalila Messaoudi, « Les délocalisations industrielles dans les mutations des systèmes productifs français ", Revue Géographique de l'Est [Online], vol. 54 / 1-2 | 2014, Online since 30 October 2014 , connection on 10 December 2020. URL : http://journals.openedition.org/rge/5153 ; DOI : https:// doi.org/10.4000/rge.5153

This text was automatically generated on 10 December 2020.

Tous droits réservés 


\title{
Les délocalisations industrielles dans les mutations des systèmes productifs français
}

\author{
Industrial relocation and the mutations in French production systems
}

Dalila Messaoudi

\section{Introduction}

1 La mise en concurrence des territoires qui accompagne le processus de mondialisation de l'économie a profondément bouleversé le paysage industriel français et la géographie de la production. En effet, certaines régions ont vu partir vers l'étranger nombre de leurs activités traditionnelles, tandis que d'autres filières sont aujourd'hui en sérieux déclin (textile, habillement, cuir, jouet, électroménager par exemple). Dans ce contexte, les délocalisations sont apparues comme particulièrement emblématiques du redéploiement mondial des activités. Sous le coup de difficultés économiques et d'une concurrence de plus en plus effrénée, mais aussi de décisions actionnariales visant à la maximisation de la rentabilité, entreprises et activités sont en quelque sorte devenues mobiles. Véritable facette de l'économie moderne, les délocalisations divisent, opposent et suscitent de vifs débats. Ces prises de position, parfois antinomiques, souvent polémiques, invitent à une analyse poussée du phénomène. Nous analyserons la participation des délocalisations aux mutations des systèmes productifs français. Nous interrogerons aussi la relation entre délocalisation et désindustrialisation. Quel est le poids des délocalisations dans la désindustrialisation $\mathrm{du}$ territoire? Quelles conséquences sur le système productif français et les territoires? 


\section{Les délocalisations : un concept multiforme, une réalité difficile à cerner}

\section{A. La délocalisation : un seul terme, mais des réalités multiples}

Lejaby en Tunisie, Majorette en Thaïlande, Valeo en Pologne, Renault au Maroc, etc.; les cas récents de transferts de production depuis la France vers l'étranger ne manquent pas. Pour autant, les délocalisations d'activités ne sont pas un phénomène nouveau. Apparue avec la crise de 1974-1975 pour désigner les mécanismes de «mobilité géographique du capital à la recherche du plus fort taux de profit ${ }^{1}$ ", l'utilisation du terme s'est généralisée en France au début des années 1990. En 1993, dans un rapport du Sénat intitulé Rapport d'information sur l'incidence économique et fiscale des délocalisations hors du territoire national, des activités industrielles et de service ${ }^{2}$, le Sénateur Jean Arthuis dénonçait fortement les conséquences économiques et sociales du phénomène en France. Mais c'est probablement l'affaire Hoover qui a donné un retentissement médiatique aux changements géographiques de production en cours, en provoquant comme un électrochoc au sein de l'opinion publique qui découvrait cette nouvelle réalité économique. En 1993, alors à la recherche de coûts de production plus avantageux, l'entreprise américaine Hoover (née en 1908) spécialisée dans la fabrication d'aspirateurs décida le transfert de ses activités de production menée en France vers l'Ecosse(Cambuslang) où le groupe originaire de l'Ohio possédait déjà une usine $^{3}$. Plus de 600 emplois ont alors été brutalement supprimés sur le site de Longvic ${ }^{4}$, près de Dijon, mettant à mal le bassin d'emplois de cette commune de 7000 habitants.

Etymologiquement, délocaliser «c'est aller hors d'un lieu». Par extension, c'est procéder à un changement de localisation. La définition la plus courante renvoie à la fermeture d'une unité de production située dans un pays A (appelé "pays d'origine »), suivie par l'ouverture d'une autre unité dans un pays B (appelé "pays de délocalisation »), qui a la charge désormais d'approvisionner le marché d'origine en biens ou en services (figure 1). Ce type de transfert est fréquemment appelé "délocalisation totale ». Les délocalisations totales d'activités sont historiquement les premières formes existantes. Ce sont probablement les opérations les plus spectaculaires mais aussi, et fort heureusement, les moins fréquentes. Leurs conséquences économiques sont souvent dramatiques, privant des villes, voire des régions entières de leur employeur principal. Ces délocalisations sont particulièrement dénoncées puisqu'elles impliquent la suppression de nombreux emplois sur le site originel. Même si elles sont très spectaculaires, les délocalisations totales sont désormais marginales en France et dans les pays industrialisés. 
Figure 1 : Schéma récapitulatif des types et des formes existantes

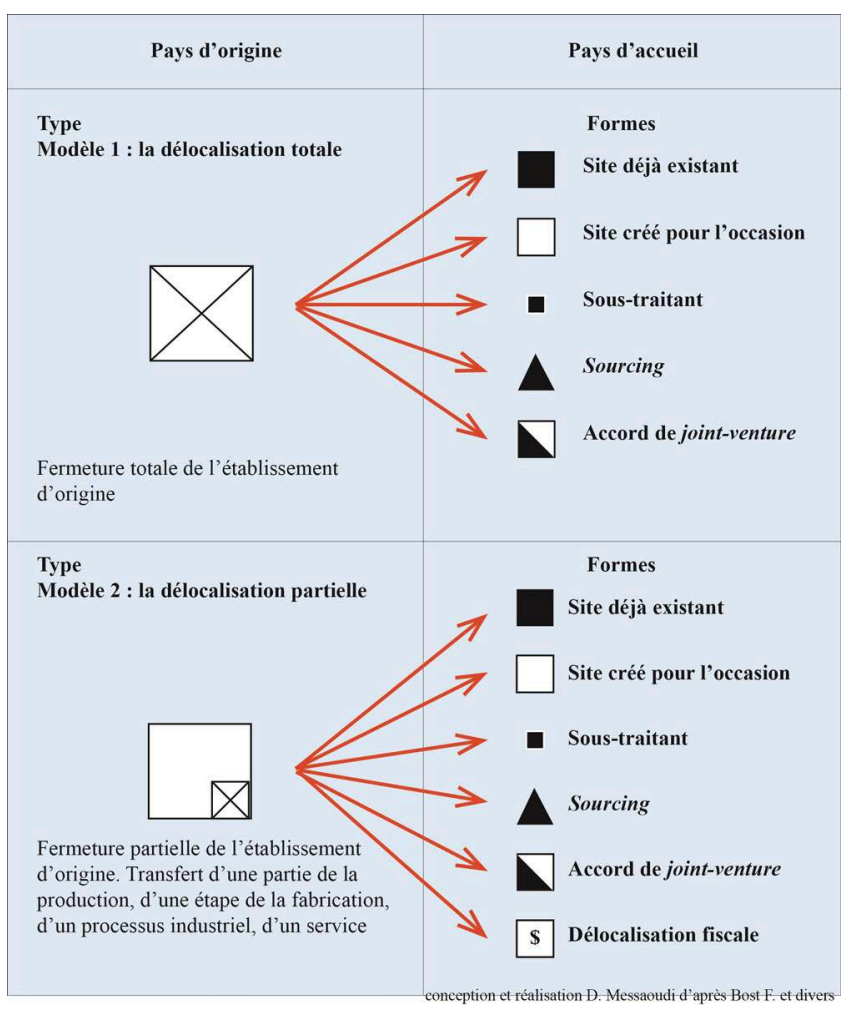

4 Les «délocalisations partielles » d'activités sont désormais plus fréquentes. Elles concernent une ou plusieurs étapes du processus de production, mais ne touchent pas l'établissement dans son ensemble. Elles sont beaucoup moins spectaculaires (il n'y a pas de fermeture de l'établissement), mais plus insidieuses (elles échappent largement aux statistiques) et à l'origine de nombreuses pertes d'emplois (généralement par grappes de quelques dizaines d'emplois).

Pour compléter ces définitions, il est important d'y inclure la notion de réimportation puisqu'elle permet de différencier les transferts stricto sensu du simple développement d'activités. D'un point de vue statistique, seul ce critère peut nous permettre de différencier les délocalisations du simple jeu du commerce international des transferts de production. Une délocalisation pourrait dès lors se définir comme toute action de transfert géographique d'une activité sous la forme d'un déplacement total ou partiel (depuis le transfert complet de l'outil de production, en l'occurrence les machines, jusqu'à la simple étape d'un process industriel) avec importation en retour de la production, entraînant la fermeture totale ou partielle de l'unité originelle, ou l'abandon en interne d'une activité particulière. En d'autres termes, il s'agit de la cessation de tout ou partie d'une activité dans le pays d'origine et de son transfert hors des frontières nationales. Lors de ce transfert, la disparition totale ou partielle d'une activité dans le pays d'origine s'accompagne soit par la création de nouveaux moyens de production dans le pays d'accueil (ouverture d'un nouvel établissement), soit par l'extension des moyens déjà existants lorsqu'il s'agit d'un établissement relevant de la même maison mère. Ce transfert peut aussi s'effectuer au profit d'un tiers qui produit à façon sous contrat (sous-traitant, équipementier), ou de fournisseurs auprès desquels il suffit de s'approvisionner lorsqu'il s'agit de biens ou de services standardisés. 


\section{B. L'approche historique ou pourquoi les transferts de production apparaissent tardivement en France}

Longtemps inférieurs à la moyenne nationale, les coûts de main-d'œuvre en province et en périphérie des grandes villes expliquent le caractère tardif des délocalisations en France. Cette manne salariale bon marché a induit, dans un premier temps, un redéploiement industriel à l'intérieur du territoire, à l'échelle nationale. On parle alors de décentralisation ${ }^{5}$. En effet, dans les années 1950-1960, afin d'aider au développement économique des régions françaises et de contrebalancer le poids excessif de la région parisienne, des aides financières ont été octroyées aux entreprises qui décidaient de redéployer une partie de leur production depuis l'Ile-de-France vers la province, et plus particulièrement vers les régions les moins développées (Bretagne, Centre, Bourgogne par exemple). Cette politique a permis une diffusion des activités industrielles puisque entre 1950 et 1975, plus de 250000 emplois ont été créés en province. Ceci est particulièrement vrai pour les industries automobile, électrique, électronique et mécanique qui ont été à l'origine de l'essentiel des emplois décentralisés.

Durant les «Trente Glorieuses », la France s'est modernisée et les transports se sont développés. L'Etat s'est voulu centralisateur, rassembleur et modernisateur. Ainsi, la réorganisation territoriale s'est fondée sur des programmes d'infrastructures lourdes. Des ports, des aéroports, des infrastructures routières et ferroviaires ont été financés. Il s'agissait en fait d'opérer une organisation territoriale par le biais de réseaux de communication, facteurs de liaison et de dynamisme. Cette période a aussi été marquée par la naissance de la Délégation à l'Aménagement du Territoire et à l'Action Régionale (DATAR) en 1963, en charge des orientations majeures du territoire français. Les initiatives publiques se sont multipliées et ont principalement concerné le secteur secondaire, vecteur de développement et créateur de richesses. L'ensemble du territoire national a donc profité du développement économique. Nous sommes dans une période de reconstruction et de satisfaction du marché national. Les entreprises sont encore très peu internationalisées et l'empire colonial constitue l'essentiel des débouchés à l'export ${ }^{6}$. A titre d'exemple, jusqu'au début des années 1970, $40 \%$ des investissements français étaient dirigés vers les seuls pays de la zone franc en Afrique (Bost, 1995, I). On peut donc dire que durant cette période, il n'y a pas eu en France de projets de délocalisation. Les marchés étaient protégés par des droits de douane et les concurrents étrangers ne pouvaient guère y pénétrer. Cette période coïncida avec une forte expansion économique pour les entreprises françaises. Néanmoins, dès la fin des années 1960, se dessinent les prémices du changement.

8 L'affaiblissement des droits de douane dans le cadre des accords du GATT, associé à la fin de l'empire colonial et à l'avènement du marché européen modifient ce schéma. En effet, l'ouverture progressive d'un grand marché où tout peut circuler, les hommes comme les marchandises, est une source de débouchés nouveaux mais aussi et surtout un facteur de concurrence. Cette nouvelle situation concoure à l'amélioration de la compétitivité des entreprises; mais elle permet en retour à de nouveaux pays de s'insérer dans le paysage économique. Les conséquences économiques de cette nouvelle concurrence sont considérables pour certains secteurs qui ne peuvent pas suivre cette dynamique (textile et habillement tout particulièrement, mécanique, etc.).

9 La question des délocalisations va réellement se poser au milieu des années 1970. Les marchés de consommation des pays industrialisés s'effondrent et les entreprises 
françaises perdent de leur compétitivité. En effet, la crise industrielle et pétrolière des années 1970 va ébranler les secteurs industriels traditionnels. Ceci est d'autant plus dramatique que ces industries sont souvent peu capitalistiques et à fort coefficient de main-d'œuvre. Le début des années 1980 marque les premières privatisations. Malgré quelques réussites (l'aéronautique par exemple), les difficultés persistent et les plans de sauvetage (acier, textile) montrent leurs limites. L'ouverture des territoires et l'environnement concurrentiel expliquent les restructurations géographiques des firmes qui se voient contraintes de repenser leurs stratégies. Cela se traduit par des pertes de dizaines de milliers d'emplois, de nombreuses fermetures de sites et le recours de plus en plus fréquent aux délocalisations. Toutefois d'autres facteurs expliquent l'avènement du phénomène de délocalisation (figure 2).

Figure 2 : Les délocalisations en France, causes et conséquences

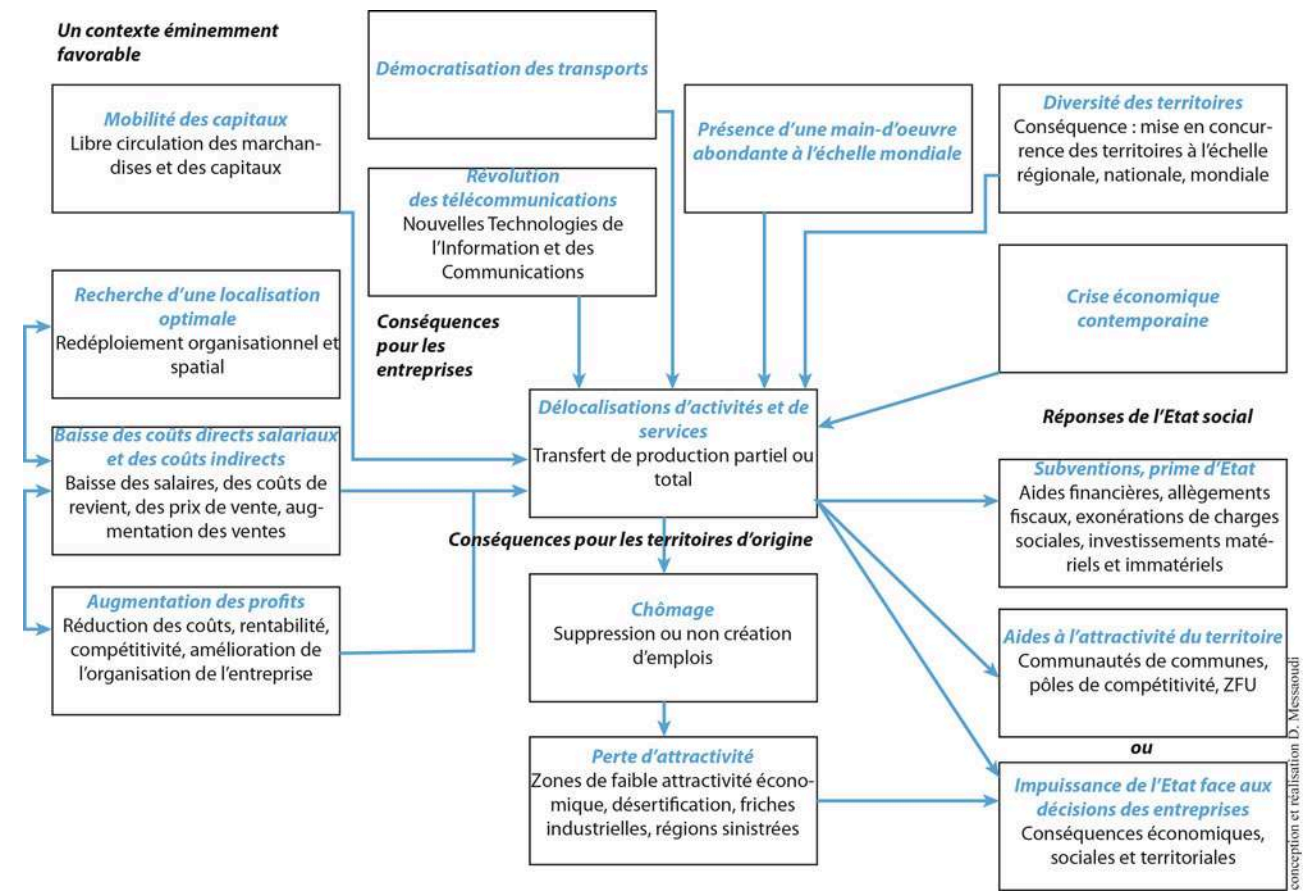

\section{Les principaux facteurs de délocalisation}

La baisse des coûts de transport et leur accessibilité grandissante ont permis la mobilité des entreprises et des sites de production. Cela a affranchi les industriels de leurs anciennes contraintes de localisation. On parle alors de " nomadisme industriel 7 ».

Un autre élément explicatif concerne les Nouvelles Technologies de l'Information et des Communications (NTIC). En effet, la révolution des télécommunications met en interconnexion croissante les acteurs du monde entier. La qualité et le coût des communications sont tels que l'on peut commencer à échanger des données avec toutes les parties du monde. Internet, les transmissions satellitaires, mais aussi les réseaux de câbles sous-marins en fibre optique tel que le South East Middle East Western Europe (SEA ME WA) facilitent les échanges. Il est dorénavant possible de disposer de liaisons fiables et peu onéreuses pour des distances très longues. Les NTlC ont donc profondément bouleversé le processus industriel classique et ont affranchi les industriels de leurs anciennes contraintes de proximité ${ }^{8}$. 
12 Le rôle de la monnaie est également fondamental. Dans le cadre de l'euro (entièrement mis en circulation le $1^{\text {er }}$ janvier 2002), les pays européens ne peuvent plus dévaluer leur monnaie afin de doper leurs exportations. De plus, le respect des règles communautaires et la logique européenne ne permettent plus à l'Etat de mener une politique industrielle autonome (le plan textile de 1996, qui accordait aux entreprises du secteur des exonérations patronales, a par exemple été refusé par la Commission Européenne).

13 La structure et les outils industriels ont aussi joué un rôle capital. La France connaît de très fortes spécialisations (énergie, ferroviaire par exemple), un secteur manufacturier plutôt spécialisé dans le moyen de gamme et très peu de PME-PMI à vocation internationale. La baisse des coûts de production devient alors, pour les entreprises, de plus en plus nécessaire. La possibilité qui s'est offerte de pouvoir segmenter la production a donc mis en évidence les disparités salariales et, par là même, a induit un nouveau type de concurrence. D'autant qu'à partir des années 1980-1990, le contexte nouveau de mondialisation entraîne une concurrence de plus en plus féroce. L'arrivée des pays « émergents » sur la scène mondiale fragilise un peu plus la compétitivité des entreprises françaises.

\section{Existe-t-il des indicateurs statistiques pertinents pour mesurer l'impact des délocalisations sur le système productif français?}

\section{A. Les indicateurs les plus utilisés pour mesurer l'impact du phénomène}

Les entreprises sont désormais de plus en plus mobiles et reconsidèrent constamment l'intérêt de leurs implantations. Pour autant, la plupart des entreprises françaises n'ont pas délocalisé la totalité de leur production mais une partie seulement. Dans ces conditions, se pose la question de la mesure du phénomène.

Une première approche statistique consiste à analyser les flux financiers et plus précisément les flux d'investissements directs à l'étranger (IDE). Les IDE peuvent être définis comme : "les capitaux que les résidents d'un pays possèdent dans un autre pays et qui sont investis prioritairement dans les activités productives par opposition aux investissements de portefeuilles ${ }^{9}$ » Ils prennent en compte le transfert de capitaux de la France vers l'étranger et peuvent prendre la forme de créations de filiales, d'extensions d'unités déjà présentes à l'étranger, de prises de participation de capital de société étrangère (à hauteur d'au moins $10 \%{ }^{10}$ ), d'offres publiques d'achat, de joint-venture, ou encore de fusions-acquisitions. Ils sont mesurables à partir des statistiques de la balance des paiements et plus précisément de la balance des capitaux. Toutefois utiliser les IDE pour mesurer l'impact des délocalisations en France est délicat. La plupart des IDE correspondent à des opérations de fusions-acquisitions. Or, seuls les rachats ou fusionsacquisitions qui conduisent les nouveaux dirigeants à réorganiser la production (fermeture de certaines unités et réouverture ailleurs) peuvent aboutir à des transferts de production. Il arrive souvent que ces rachats n'entraînent pas de délocalisation (figure 3). On peut donc dire que l'essentiel des IDE sert à conquérir de nouveaux 
marchés et non à remplacer une production française par une production à bas coûts de main-d'œuvre.

Tableau 1 : Forme d'IDE menant ou non à une délocalisation

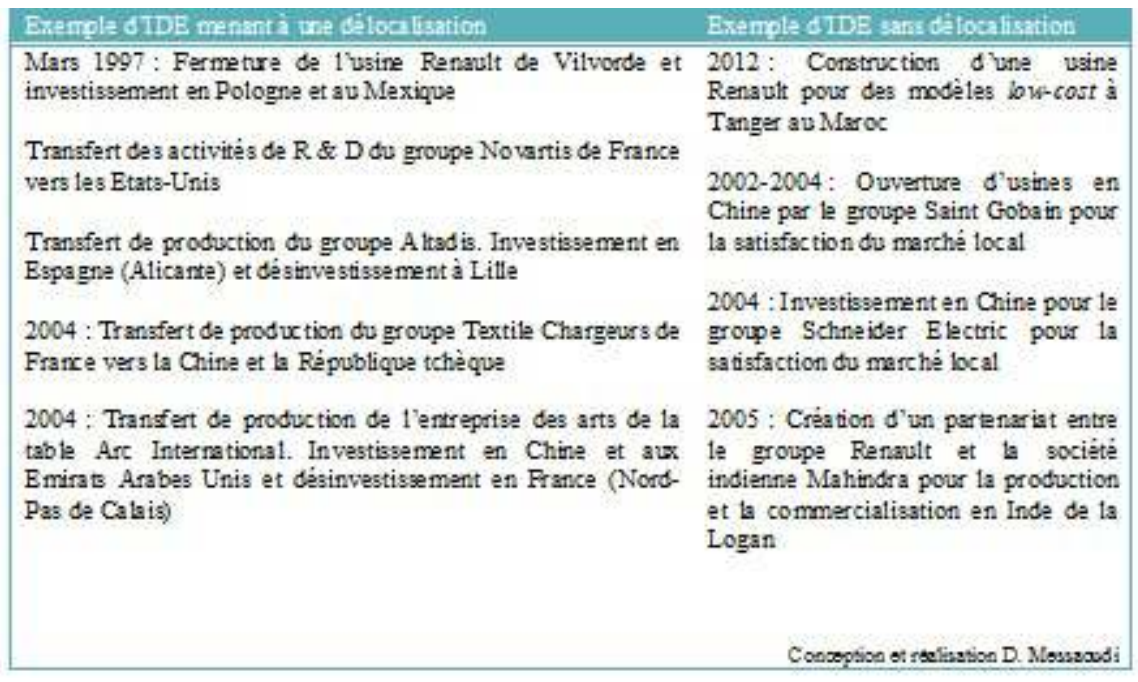

Une autre approche consiste à analyser les flux d'importations grâce aux données douanières. Néanmoins, toutes les baisses d'exportations et les hausses d'importations ne peuvent être imputées aux seules délocalisations compte tenu de nombreux autres facteurs (perte de compétitivité ou dégradation suite à un effet de change; hausse de l'Euro sur le Dollar engendrant une baisse des exportations).

Un autre moyen consiste à s'appuyer sur des données tirées du répertoire SIRENE d'établissements et d'entreprises (Système Informatique pour le Répertoire des Entreprises et leurs établissements) et sur les sources que peuvent fournir indépendamment les entreprises et les groupes. Mais utiliser les données d'entreprises reste délicat. Les transferts de production sont un sujet éminemment sensible et les industriels n'aiment guère communiquer sur leurs transferts de production. D'abord que parce liées à l'emploi, les délocalisations inquiètent. On ne saurait nier les stratégies d'entreprise dont la survie passe parfois par ce transfert de production. Maisil arrive aussi que certaines décisions relèvent plus de l'anticipation que du non choix. Elles sont alors justifiées par la rentabilité ; non pas que ces établissements ne soient pas rentables mais ils ne le sont pas suffisamment au regard des investisseurs. Ces opérations ne sont pas comprises par les salariés, les cadres et l'opinion publique. C'est pour cela que l'on assiste souvent à une politique de fait accompli. Les répercussions pour une entreprises qui délocalise peuvent être lourdes de conséquences (sur le cours des actions, la réaction des élus locaux, les syndicats, par exemple). Cela limite la transparence de l'information.

L'analyse chiffrée du problème est donc bien difficile. Aucune donnée n'est capable de mesurer précisément l'impact des délocalisations et d'apprécier le phénomène dans son ensemble. Plusieurs études ont, néanmoins, tenté de mesurer les retombées en matière d'emplois. 


\section{B. Stratégie de développement ou de destruction d'emplois ?}

19 Sur la question des délocalisations et des retombés en matière d'emplois, il existe deux familles de pensée dominantes chez les économistes: les «positivistes» et les « relativistes".

Les «positivistes» considèrent que les pertes d'emplois liées aux transferts de production seraient faibles et fortement compensées par les gains réalisés en termes de baisse de prix et de nouveaux débouchés (Cohen 2006, Amiti et Wei 2005). En effet, les délocalisations permettent de bénéficier de coûts de main-d'œuvre avantageux, qui réduisent les prix de revient et de vente et qui se répercutent sur les budgets de consommation. Au début du XIXe siècle, David Ricardo dans son ouvrage Essai sur l'influence des bas prix du blé sur les profits du capital ${ }^{11}$ expliquait déjà que la baisse des prix des céréales permise par leur production à l'étranger permettait de réduire les coûts salariaux et donc de favoriser l'industrie. Aujourd'hui encore, un grand nombre d'analyses économiques considèrent les délocalisations comme un élément de compétitivité qui permet aux entreprises de maintenir leur position concurrentielle. Xavier Mosquet, économiste et Directeur associé du Boston Consulting Group (BCG), a réalisé en 2004 une étude auprès de 16 grands groupes français ayant procédé à des déplacements de production ou ayant développé leur activité à l'international. Après avoir étudié l'évolution du chiffre d'affaires et de l'emploi de ces grands groupes entre 1995 et 2003, il arrive à un constat surprenant: ces entreprises, qui auraient dû selon toute attente supprimer des emplois en France ${ }^{12}$, ont finalement maintenu voire augmenté leurs effectifs français. Selon l'auteur, c'est l'activité réalisée hors de France qui a créé des emplois: "en participant à la mondialisation, ces entreprises ont crée plus d'emplois que si elles étaient restées sur leur marché national ». Une autre étude réalisée par l'Assemblée des chambres françaises de commerce et d'industrie montrait qu'en 2004, les délocalisations ont été à l'origine de 10000 emplois perdus contre 30000 créés par des investissements étrangers en France. Il y avait donc à cette date plus d'emplois créés du fait d'investissements étrangers que d'emplois perdus suite à des transferts de production $^{13}$.

21 Selon ces analyses, agir mondialement serait donc un moyen de créer de l'emploi localement et les pertes d'emplois liées aux délocalisations pourraient être compensées par des gains en termes de compétitivité ou par de nouvelles embauches à d'autres échelles. Cette famille de pensée côtoie un autre grand courant qui, sans parler d'impact positif, relativise la portée du phénomène.

L'analyse la plus pertinente est probablement celle effectuée par Patrick Aubert et Patrick Sillard ${ }^{14}$. Ces auteurs ont tenté de repérer les transferts de production en comparant la baisse des emplois dans un secteur donné suivi d'une hausse des importations. Les délocalisations sont détectées lorsque l'emploi diminue ou disparaît au sein d'un établissement et que les importations du groupe détenant l'établissement touché augmentent pour le type de biens auparavant produits en France. Ainsi, en comparant l'évolution de l'emploi délocalisé et les taux de chômage par zone, les auteurs tentent de démontrer que les délocalisations n'ont qu'un effet limité sur les pertes d'emplois ${ }^{15}$. Ils estiment ainsi qu'entre 1995 et 2001, 95000 emplois ont été supprimés suite aux délocalisations (sur un total de 500000 emplois supprimés en France). 
Une autre étude INSEE, réalisée sur la période 1970-2002, montre que seulement $10 \%$ des emplois industriels détruits font suite à des transferts de production en France ${ }^{16}$. Plus récemment, sur la période 2000-2005, les délocalisations à l'étranger auraient généré approximativement 36000 destructions d'emplois par an soit $4 \%$ de l'emploi des sociétés ayant délocalisé et 20000 destructions d'emplois entre 2009 et $2011^{17}$.

Une majorité des études qui ont tenté d'évaluer l'impact réel des délocalisations sur la destruction des emplois en France insiste sur l'impact limité ou contenu des délocalisations dans les pertes d'effectifs industriels totales. Pourtant le retentissement médiatique exceptionnel de certaines fermetures d'usines exacerbe la portée du phénomène.

\section{Les délocalisations : un fort retentissement médiatique mais une contribution limitée dans la désindustrialisation des territoires}

25 Même si elles sont relativement faibles au niveau national (quelques centaines d'emplois par exemple), les conséquences d'une fermeture d'usine n'en sont pas moins dramatiques à des échelles plus fines, si elles touchent des bassins d'emplois fragiles. En effet, le principal enjeu lié aux déplacements d'activités est l'impact sur l'économie et l'emploi, car le schéma classique veut que l'on transfère dans les pays à bas salaires les activités à fort coefficient de main-d'œuvre. En conséquence, les travailleurs les moins qualifiés (de loin les plus nombreux) sont plus exposés. Si les transferts s'accompagnent de fermetures d'usines sur les sites originels, ils peuvent être à l'origine de nombreuses pertes d'emplois. Entre 2002 et 2010, 700000 emplois industriels ont ainsi été détruits en France passant de 4,3 à 3,6 millions de salariés au total $(-16 \%)^{18}$. A l'échelle mondiale, l'évolution est tout autant significative. Depuis une trentaine d'années, les pays anciennement industrialisés ont vu leurs effectifs industriels diminuer tandis que ceux de certains pays émergents ont augmenté. Ainsi, la Chine a créé plus de 30 millions d'emplois dans l'industrie manufacturière entre 2000 et 2008 suivie par le Mexique et le Brésil ${ }^{19}$. Il est donc facile d'établir un lien direct entre les délocalisations et la désindustrialisation en France. Pourtant, si certaines fermetures d'usines sont effectivement des délocalisations, d'autres correspondent plutôt à des réorganisations de production ou à des dépôts de bilan (Boussac, DMC par exemple). D'autres éléments peuvent également expliquer la baisse des effectifs industriels. Entre 1993 et 2008, la production industrielle française a augmenté alors que dans le même temps, les effectifs ont diminué. Cette hausse de production industrielle est consécutive aux évolutions technologiques et aux gains de productivité. Ainsi, selon un rapport de la Direction générale du trésor et de la politique économique (DGTPE), la hausse de productivité expliquerait la suppression de $30 \%$ des emplois industriels français entre sur la période 1980-2007 et de $65 \%$ depuis $2000^{20}$. De plus, les dénominations d'activités industrielles et de services tendent à s'entrecroiser. L'externalisation et le recours à la sous-traitance ont transformé certains métiers industriels en services. Une partie des activités externalisées des entreprises comme la $R$ \& D ou le marketing est comptabilisée en tant que fonction tertiaire, même si la maison mère a une activité de production manufacturière. Ce transfert d'activités représenterait, selon une étude de la DARES, $30 \%$ des suppressions d'emplois industriels ${ }^{21}$. La part des emplois intérimaires est également à prendre en compte. Souvent enregistrées statistiquement comme relevant du tertiaire, ces activités appartiennent le plus souvent au secteur 
secondaire. Les limites entre les secteurs sont en effet de plus en plus difficiles à établir ${ }^{22}$. D'autres facteurs sont également à l'origine de la destruction des emplois industriels en France et ont fait l'objet de nombreuses analyses: un secteur manufacturier plutôt spécialisé dans le moyen de gamme et très peu de PME-PMI à vocation internationale, le manque de compétitivité, l'insuffisance de l'investissement dans la $\mathrm{R} \& \mathrm{D}$ et dans l'innovation, un marché domestique saturé et un marché européen en berne, l'environnement financier, fiscal, institutionnel, le marché communautaire et les règles de non distorsion, la montée en puissance des pays émergents, etc. (Artus 2011, Krugman 2000).

26 La désindustrialisation ne résume donc pas simplement à la fermeture de sites et le transfert à l'étranger. La désindustrialisation est le résultat de l'interaction de plusieurs facteurs dont les délocalisations ne sont que l'une des causes, mais le retentissement médiatique de certaines fermetures d'usines les rend particulièrement visibles.

\section{Des conséquences territoriales souvent spectaculaires}

\section{A. Des mutations structurelles et organisationnelles}

Les entreprises cherchent désormais à gérer leurs activités de manière optimale en jouant aussi bien sur l'organisation de leur processus productif à l'échelle mondiale que sur la localisation de leurs activités, souvent au plus près des territoires. Elles sont à la recherche d'une plus grande "rationalisation de production ${ }^{23}$ " et veulent tirer parti des avantages comparatifs en se localisant de manière optimale, qu'il s'agisse de coûts ou de compétences. Cela suppose que les tâches et les activités soient auparavant segmentées pour être plus facilement transposables ailleurs. En effet, la fragmentation des processus productifs permet d'offrir de nouvelles opportunités de localisation et modifie la concentration géographique des activités. Le produit fini est décomposé en sous-produits ce qui permet une différenciation géographique des tâches de production. Cette segmentation aboutit à terme à la « Décomposition Internationale du Processus Productif» (Lassudrie-Duchêne Bernard 1982, Fontagné 1991, Mouhoub 1993). Les tâches sont d'abord décomposées d'un point de vue technique pour faciliter leur reproduction. Elles sont ensuite fragmentées d'un point de vue spatial pour que l'on puisse transposer la production. L'enjeu principal est que, dès lors que cette fragmentation est possible, les entreprises peuvent procéder à une standardisation de leur production. Plus un produit est standardisé, plus sa reproduction est facilitée et plus le coût de la main-d'œuvre est faible. Dans ce contexte, les entreprises ont développé des Réseaux Internationaux de Production (RIP) ${ }^{24}$ réduisant par là même le concept de frontière. La compétition se joue alors à toutes les échelles et l'attraction ou le maintien des RIP devient un enjeu territorial primordial. Tous les pays peuvent désormais être mis en concurrence sur chaque élément du processus productif. En atteste les «fabless ${ }^{25}$ " autrement appelées les « industries sans usine » qui ne gardent en leur possession que les activités de conception, de R\&D et délocalisent le reste. C'est le cas de l'entreprise américaine Dell qui commercialise des ordinateurs et des produits informatiques mais qui achète tous les composants de ses produits à des sous-traitants étrangers. ATI Technologies, spécialisée dans les puces graphiques pour les ordinateurs fonctionne également sur le même principe. 
28 La fragmentation de la chaîne de valeur a donc modifié durablement la répartition géographique du tissu productif. Mais d'autres éléments ont induit de profondes mutations organisationnelles. Dans l'industrie de l'habillement par exemple, les formes d'approvisionnement ont profondément changé. Le schéma qui prévalait il y a quelques années peut se résumer de la façon suivante: le donneur d'ordres achetait un tissu français ou étranger. Il l'exportait dans le pays de délocalisation (Maroc, Tunisie par exemple) accompagné du patronage pour faire confectionner le modèle et le réexpédier ensuite en France. C'est ce que l'on appelait le Trafic de Perfectionnement Passif (TPP). Mais face aux difficultés, les chaînes spécialisées, comme les grandes marques cherchent à réduire leurs coûts. La cotraitance va alors faire son apparition. Le confectionneur ne se contente plus d'être un acteur passif. On lui demande désormais une participation active en fournissant les matières premières (tissu, fils etc.). Par la suite, le recours au sourcing international commence à s'imposer.Appelé aussi négoce international, il consiste à acheter à l'étranger des produits semi-finis ou finis pour son propre marché. Le modèle est choisi au sein d'une collection préexistante et les distributeurs y apposent leur griffe. C'est un mode d'approvisionnement qui a connu une ascension fulgurante ces dix dernières années. La première conséquence est que la vague de délocalisations entamée dans le secteur de l'habillement dès les années 1970 s'intensifie et se propage aux activités textiles situées en amont et considérées jusquelà comme protégées parce que plus techniques. L'ensemble de la filière s'est reconstituée progressivement à l'étranger: tisseurs, filateurs, confectionneurs, sont désormais tous présents dans les mêmes zones géographiques.

L'exemple de l'entreprise française de prêt à porter Jennyfer témoigne de cette évolution. C'est en 1986 que s'ouvre la première boutique Jennyferà Saint-Denis. L'approvisionnement est très concentré. La production d'articles maille (pulls, teeshirt, etc.) provient essentiellement d'Afrique du Nord (Maroc et Tunisie), le reste étant assuré par des fabricants français (Clamart). La production d'articles chaîne et trame (pantalons, robes, chemisiers, grosses pièces, etc.) suit approximativement le même schéma. La majeure partie de la production est effectuée par des confectionneurs nordafricains (Maroc et Tunisie), le reste étant assuré par des confectionneurs français (Région Rhône-Alpes et Nord-Pas-de-Calais). Les articles «tests » et les petites séries sont réalisés dans les ateliers du Sentier parisien afin de bénéficier de délais très courts.Toutefois la fin des Accords Multifibres, devenus Accords sur les Vêtements et les Textiles (ATV) en 1994, modifie la donne. Les ATV permettaient de conclure des accords bilatéraux de restrictions d'importations de certaines catégories de produits textiles et d'habillement. En 1986, on comptait ainsi plus de 81 accords de restrictions et plus de 1000 contingentements. Depuis le $1^{\mathrm{er}}$ janvier 2008, il n'existe plus de quotas d'importation. L'Asie et surtout la Chine proposent désormais des produits concurrentiels sans limitation de volume. La Chine s'est en effet démarquée très précocement de ses concurrents par la faiblesse de ses coûts de main-d'œuvre, la solidité de ses infrastructures logistiques et ses efforts d'investissement 
Figure 3 : Modalité organisationnelle de production et d'approvisionnement de l'entreprise Jennyfer entre 2000 et 2010

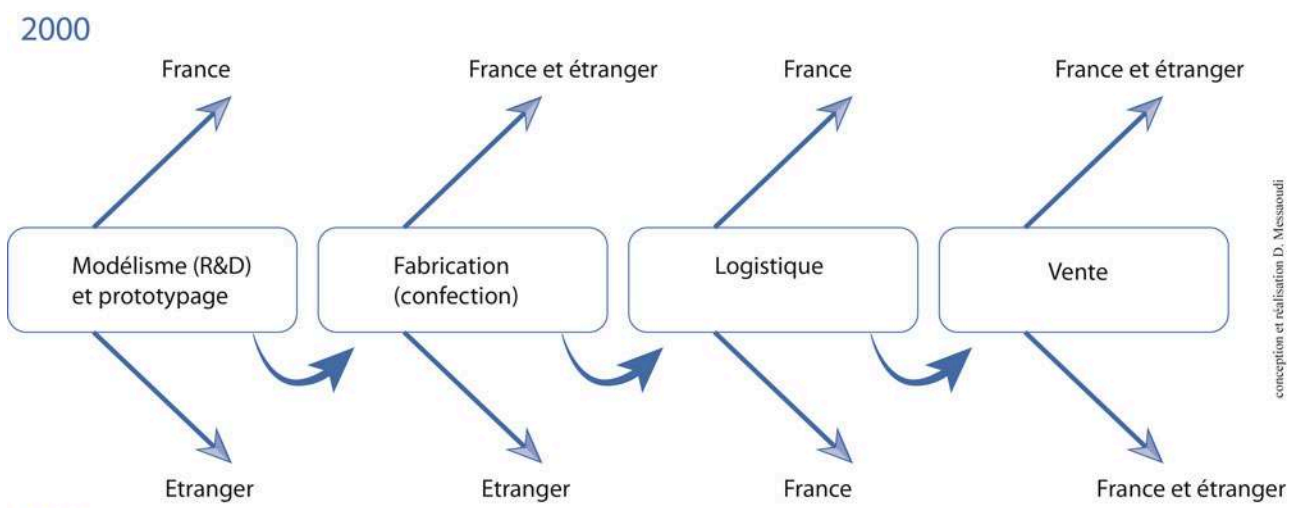

Depuis 2006, l'entreprise ne réalise plus ses prototypages (montage d'un modèle type) ni son modélisme en France (figure 4). Ces activités sont désormais réalisées à l'étranger. $80 \%$ de la production provient désormais de la Chine et est réalisée sous forme de produits finis ${ }^{26}$.L'exemple de l'entreprise Jennyfer n'est pas un cas isolé. Ce changement organisationnel a touché lieu en premier la grande distribution qui a obligé, indirectement, de nombreuses autres entreprises à faire de même. Il touche aujourd'hui les chaînes et magasins spécialisés.

31 Toutefois les mutations les plus spectaculaires et les plus visibles concernent les paysages. En effet, l'usine a structuré pendant longtemps les paysages et les représentations.

\section{B. De fortes transformations paysagères}

Jusque dans les années 1970, les villes industrielles étaient indissociables et indissociées de l'activité de production dominante : qu'il s'agisse de Peugeot à Sochaux, de Michelin à Clermont-Ferrand ou encore des chantiers navals de Saint-Nazaire. De l'image de la ville productrice comme de la stabilité économique découlaient une cohésion sociale et une identité collective. A l'image du Creusot, la ville-usine s'est structurée autour de l'outil de production et la ville industrielle a grandi, profitant de capitaux financiers, mais aussi des capitaux sociaux que constituent les savoir-faire. Les fumées des usines sont alors le témoin heureux de l'activité économique intense. L'usine devient facteur de solidarités ouvrières, d'identité, mais aussi de structures spatiales particulières. On peut ainsi reprendre les mots du poète Félix Leclerc (Leclerc 1964) «La ville ? C'est le peuple rassemblé autour des usines ». On voit bien ici le couple indissocié que créent alors l'usine et la ville et avec elles les salariés.

Cette étroite relation territoires/entreprises a eu de profondes et graves conséquences sociales et économiques à partir des années 1970-1980. Pierre Veltz parle d'un processus de « déliaison entre le territoire et l'industrie » (Veltz, 1999) qui s'est amorcé dès la période des Trente Glorieuses comme une phase suivant l'époque du paternalisme. Ainsi, les nouvelles logiques mises en œuvre aboutissent de façon quasisystématique à une rupture des liens géographiques entre les sites, leurs sous-traitants et les fournisseurs et, conséquemment, entre les lieux décisionnels et les salariés. A l'image de Valenciennes des années 1990, des villes se sont vidées de leur substance industrielle affichant des panneaux de vente aux fenêtres. Le paysage de ces régions 
industrielles subit alors de violents traumatismes. Les cheminées, les terrils, les chevalements de mine, qui se dressaient en portant la fierté de l'ensemble d'une société industrieuse et productive, hérissent alors des paysages vides de ces activités économiques. Le traumatisme paysager est autant environnemental que psychologique et s'inscrit durablement dans les représentations collectives et l'image portée.

Les friches industrielles sont très certainement l'élément le plus visible de ce paysage (photo 1). A l'abandon ou en conversion, elles se localisent de façon plus importante en Lorraine, dans le Nord-Pas-de-Calais et en Région parisienne (Terril de Loos-en-Gohelle, anciennes usines textile de Roubaix par exemple). En effet, le départ des industries a laissé la place à des friches ou des dents creuses, qui nécessitent parfois des travaux de dépollution importants avant leur réaffectation et leur requalification ${ }^{27}$. La géographie des relations entre l'espace urbain et l'espace usinier se modifie à mesure que les conséquences de l'internationalisation des entreprises s'impriment dans la ville. La quasi identification de la ville industrielle à son entreprise n'est plus de mise et les villes sont parfois doublement traumatisées : par la perte d'emplois, mais aussi par les conséquences écologiques. Ainsi, à Auboué, en Meurthe et Moselle, la cité ouvrière se fissure en raison des mouvements de terrains causés par les galeries souterraines creusées au moment de l'exploitation minière.

Photo 1 : Usine Forgeval à Valenciennes fermée en 2001

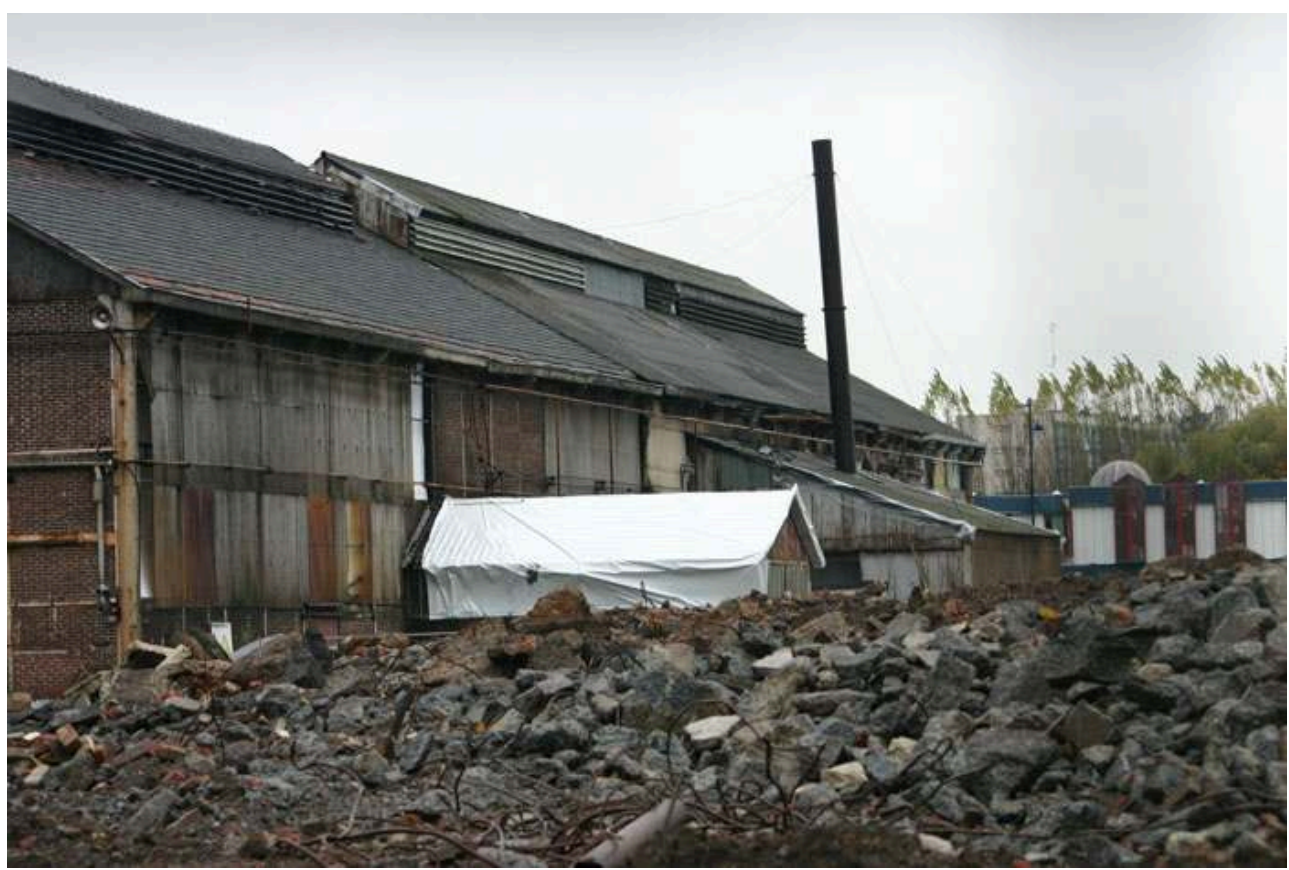

(C) C. Luxembourg

Mais la ville industrielle n'a pas pour autant disparu. Elle reste industrielle par la forme d'habitat, par la volonté politique de conserver, de patrimonialiser, par son identité culturelle et puis parfois encore par ses activités économiques. Elle côtoie désormais de nouveaux espaces industriels. En effet, jusqu'aux années 1980, la géographie des coûts a déterminé les premières localisations. Depuis les années 1990, la géographie des compétences s'est progressivement imposée comme un facteur de localisation déterminant laissant apparaître de nouveaux espaces d'accueil et de développement des activités économiques. 


\section{Les mutations de l'espace industriel et les nouveaux espaces d'accueil : l'exemple des pôles de compétitivité} retour de la " politique industrielle ». Celle-ci avait été jusque-là largement évincée des préoccupations politiques et d'aménagement du territoire au nom des changements de paradigmes propres à l'idée de société dite "post-industrielle ", où la composante industrielle à proprement parler n'avait plus guère sa place face à la tertiairisation de l'économie. De plus en plus d'observateurs, de décideurs et d'hommes politiques militent désormais pour l'élévation de la réindustrialisation du territoire national au rang de priorité majeure. L'idée est donc, à travers toute une série de mesures, d'inciter à la ré-industrialisation et à la promotion de l'emploi industriel, en misant notamment sur l'innovation, sur tout le territoire et à long terme. En effet, les nouvelles formes d'industrie sont désormais davantage fondées sur des produits à plus forte valeur ajoutée que sur une main-d'œuvre nombreuse. Les industriels français doivent donc s'adapter en se réorientant sectoriellement ou en développant des activités de recherche-développement. Les partenariats constituent aussi une dimension essentielle, d'abord entre les acteurs privés et publics, mais aussi entre l'industrie et la recherche et entre les PME et les PMI. C'est le cas des pôles de compétitivité qui rassemblent des entreprises concurrentes ou / et complémentaires.

Par définition, un pôle de compétitivité est un lieu de rencontres qui met en relation sur un même territoire (pas forcément continu) des entreprises, des centres de formation et des unités de recherches publiques et privées afin d'élaborer des projets communs de développement et d'innovation. C'est donc un regroupement d'acteurs dans un lieu donné et un moyen d'augmenter la productivité et le rayonnement attractif d'un réseau d'entreprises et d'acteurs sur une base territoriale. Aux Etats-Unis, Michael Porter ${ }^{28}$, professeur à l'université de Harvard, a mené d'importants travaux sur le concept des clusters. En France, c'est en 2004 que le gouvernement Raffarin a décidé d'encourager la création d'une quinzaine de pôles de compétitivité dans les hautes technologies et les activités classiques en accordant des avantages fiscaux. Mais, déjà dans les années 1990, la DATAR avait posé les prémices du phénomène en valorisant le concept de Systèmes Productifs Locaux ou SPL (sur le modèle italien), en tant qu'organisation en réseau. Ces SPL se sont généralement spécialisés dans des secteurs bien précis (Mecanic Valley, etc.). En 2005, le Comité Interministériel d'Aménagement et de Compétitivité des Territoires (CIACT) a annoncé la création de 67 pôles de compétitivité, dont 15 mondiaux ou à vocation mondiale sélectionnés à partir de trois critères fondamentaux : l'existence d'un partenariat science-industrie, la référence à un marché et/ou une technologie. En 2011, et après une seconde vague de création, on compte 71 pôles de compétitivité. Le financement est effectué par le biais des ministères, ainsi que par la Caisse des Dépôts, l'Agence Nationale de la Recherche (ANR), l'OSEO (fusion de l'ANVAR et de la BDPME) et l'Agence de l'Innovation Industrielle (fusionné à l'OSEO en 2007) ${ }^{29}$. Sur les 71 pôles identifiés, on compte 7 pôles mondiaux et 10 pôles à vocation mondiale. Les choix d'implantation des pôles de compétitivité ont permis une mise en commun des compétences. Les élus des régions Aquitaine et Midi-Pyrénées ont, par exemple, décidé de travailler ensemble afin d'accueillir "l'Aerospace Valley ", un pôle de compétitivité dédié à l'aéronautique, aux activités spatiales et aux systèmes embarqués ${ }^{30}$. L'Aerospace Valley est construit autour

Revue Géographique de l'Est, vol. 54 / 1-2 | 2014 
d'un Aerospace campusqui regroupe plus de 75 grandes entreprises, 250 PME, toutes les grandes écoles aéronautiques de Toulouse et accueille les chercheurs de l'office National d'Etudes et de Recherches Aérospatiales (ONERA). Ces deux régions sont complémentaires puisque l'Aquitaine est plutôt spécialisée vers l'aéronautique militaire et d'affaires et la région Midi-Pyrénées vers l'aéronautique civile.

Même si le bilan est à nuancer (en termes de lisibilité et de gouvernance en particulier), l'objectif affiché des pôles est de rendre les territoires compétitifs et attractifs. Ils témoignent d'un certain retour de la "politique industrielle » sur le devant de la scène et ont pour vocation de maintenir l'activité en France.

\section{Conclusion}

Sous le coup de difficultés économiques et d'une concurrence de plus en plus effrénée, mais aussi de décisions actionnariales visant à la maximisation de la rentabilité, entreprises et activités sont en quelque sorte devenues mobiles, ce qui entraine de profonds bouleversements dans les territoires de départ mais aussi d'arrivée. Car, même si elles sont relativement faibles au niveau national, les conséquences d'une délocalisation n'en sont pas moins dramatiques à des échelles plus fines, si elles touchent des bassins d'emplois fragiles. Ces conséquences peuvent être directes et visibles, mais aussi indirectes et plus difficilement perceptibles sur les sous-traitants, les transporteurs, les fournisseurs. Finalement, toute l'économie d'une région peut être atteinte si la situation financière des familles touchées par ces licenciements se répercute par exemple sur l'activité des commerces en général. Les délocalisations ont donc conduit à des mutations profondes des systèmes productifs entendues dans ses dimensions spatiales, organisationnelles et économiques. Pourtant leur part dans la désindustrialisation $\mathrm{du}$ territoire est plus limitée qu'il n'y parait. La désindustrialisation est le résultat de l'interaction de plusieurs facteurs dont les délocalisations ne sont que l'une des causes mais non l'origine principale. Le retentissement médiatique des fermetures d'usines, les articles ou les discours politiques nous rappellent régulièrement que cette réalité existe mais elle reste finalement moins spectaculaire qu'il n'y parait. L'intérêt de cette réflexion a été de replacer la question des délocalisations dans les mutations des systèmes productifs et dans la désindustrialisation du territoire en s'apercevant de son rôle autrement moins important que celui de l'internationalisation des entreprises et du redéploiement mondial des activités.

\section{BIBLIOGRAPHY}

Arthuis J., 1993, Rapport d'information sur l'incidence économique et fiscale des délocalisations hors du territoire national, des activités industrielles et de service, Rapport n³37, Sénat, $231 \mathrm{p}$. 
Artus P., 1995, « Délocalisations industrielles, croissance et bien-être », Économie appliquée, vol. XLVIII, $\mathrm{n}^{\circ} 1$, janvier, p.31-46.

Artus P., Virard M-P., 2011, La France sans ses usines, Paris, Fayard, 171 p.

Askenazy P., 2012, « Faut-il craindre la désindustrialisation? », Problèmes économiques, nº 3040, p. $35-40$.

Aubert P., Sillard P., 2005, Délocalisations et réductions d'effectifs dans l'industrie française, Document de travail de la DESE, Paris, Insee, $47 \mathrm{p}$.

Auray J.P., Bailly A., Derycke P.H., Huriot J.M., 1994, Encyclopédie d'économie spatiale. Concepts, comportements, organisations, Paris, Economica, $427 \mathrm{p}$.

Aydalot P., 1985, Economie régionale et urbaine, Paris, Economica, 487 p.

Bailoni M., 2009, « Le Patrimoine dans le renouveau d'une région postindustrielle, l'exemple du Nord-Est anglais », Revue Géographique de l'Est, vol.48, $\mathrm{n}^{\circ} 1 / 2$.

Battiau M., 1998, L'industrie, définition et répartition mondiale, Paris, SEDES, Campus Géographie, 191 p.

Benko G., Lipietz A., 2000, La richesse des régions. La nouvelle géographie socio-économique, Paris, PUF, $564 \mathrm{p}$.

Bonnet J., Broggio C., 2009, Entreprises et territoires, Paris, Ellipses, 238 p.

Bost F., 2012, Entreprises et territoires à l'épreuve de la mondialisation de l'économie, Habilitation à diriger des recherches, Université de Paris-Ouest-Nanterre-La Défense, 369 p.

Bost F., Delapierre M., 2007, « Réseaux internationaux de production et reconfiguration des territoires ", Economies et sociétés, Série Relations économiques internationales, n 38, p.237-254.

Bouba-Olga O., 2006, Les nouvelles géographies du capitalisme. Comprendre et maîtriser les délocalisations. Paris, Seuil, 240 p.

Carre D., Levratto N., 2013, Les entreprises du secteur compétitif dans les territoires. Les déterminants de la croissance, Etude de l'Assemblée des communautés de France (ADCF), 80 p.

Carroue L., 2013, La France. Les mutations des systèmes productifs, Paris, Armand Colin, 235 p.

Colletis G., 2012, L'urgence industrielle, Paris, Le bord de l'eau, 200 p.

Conférence nationale pour l'industrie, 2011, Ensemble réindustrialiser la France pour la croissance et l'emploi, $274 \mathrm{p}$.

DATAR, 2010, Territoires 2040. Aménager le changement, Paris, La documentation Française, 103 p.

DATAR, 2006, 40 ans d'aménagement du territoire, Paris, La Documentation française, 158 p.

Davezies L., 2012, La crise qui vient, Paris, Le Seuil, 128 p.

Daviet S., 2005, Industrie, culture, territoire, Paris, L'Harmattan, 208 p.

Deshaies M., 2007, Les territoires miniers, exploitation et reconquête, Paris, Ellipses, 224 p.

Demmou L., 2010, La désindustrialisation en France, Document de travail, Direction générale du trésor et de la politique économique (DGTPE), $49 \mathrm{p}$.

Dicken P., 2003, Global Shift: Reshaping the Global Economic Map in the 21st Century, London, Sage, 632 p. 
Dunning J-H., 2002, Regions, Globalization and the knowledge-based economy, Oxford, Oxford University Press, 506 p.

Echaudemaison C.D., 1989, Dictionnaire d'économie et de sciences sociales, Paris, Nathan, p.143.

Edelblutte S., 2009, Paysages et territoires de l'industrie en Europe : héritages et renouveaux, Paris, Ellipses, $272 \mathrm{p}$.

Fache J., 2006, Les mutations industrielles, Paris, Belin, 190 p.

Fontagné L., Lorenzi J.H., 2005, Désindustrialisation et délocalisation. Conseil d'analyse économique, $400 \mathrm{p}$.

Fontagné L., D'isanto A., 2013, Chaînes d'activités mondiales : Des délocalisations d'abord vers l'Union européenne, INSEE Première, $\mathrm{n}^{\circ} 1451,4 \mathrm{p}$.

Gallois L., 2012, Pacte pour la compétitivité de l'industrie française, Paris, La documentation Française, $67 \mathrm{p}$.

Geneau de Lamarlière I., StaszakJ-F., 2000, Principes de géographie économique, Paris, Bréal, Grand Amphi Géographie, 448 p.

Giraud P-N., Weil T., 2013, L'industrie française décroche-t-elle? Paris, La documentation Française, $190 \mathrm{p}$.

Grou P., 2005, Impératif technologique ou déclin économique, Paris, l'Harmattan, Questions Contemporaines, $132 \mathrm{p}$.

Hatem F., 2005, L'attractivité du territoire : de la théorie à la pratique, Ministère de l'Economie et des Finances, Service des Etudes et des Statistiques Industrielles, p.269-283.

Krugman P., 1991, « Increasing return and economic geography», Journal of Pol, Economy, n 99.

Laine F., 2005, Secteurs et métiers industriels : l'industrie n'est plus ce qu'elle était, Premières synthèses et informations de la DARES, Ministère de l'Emploi, du Travail et de la Cohésion sociale, $\mathrm{n}^{\circ} 16.2$.

Lassudrie-Duchene B., 1982, Décomposition internationale des processus productifs et autonomie matinale, In BOURGUINAT H, Paris, Economica, p.45-56.

Le Blanc G., 2012, « Dynamiques des territoires industriels. Essai de prospective dans le cadre de Territoires $2040 »$, Futuribles, n 385, p.24.

Leriche F., Zuliani J-M., 2003, « Airbus et les recompositions territoriales à Toulouse et Bristol », Géographie et Cultures, n 48, p.61-80.

Luxembourg C., 2008, Villes en transition : les avatars de l'industrie dans les villes françaises petites et moyennes (Blagnac, Bourges, Gennevilliers, Le Creusot, Valenciennes), Thèse de doctorat, Géographie, Université Paris-Ouest-Nanterre-La Défense, 336 p.

Merenne-Schoumaker B., 1998, «Les délocalisations industrielles. Pour une clarification d'un phénomène mal connu et controversé ", in Wakerman G., Nouveaux espaces et systèmes urbains, Paris, SEDES, p.257-267.

Michalet C.A., 2005, L'impact en Europe des délocalisations vers les pays méditerranéens, Note et études Anima n8, Agence Française pour les Investissements Internationaux, 145 p.

MOUHOUB E.M., 1992, Changement technique et division internationale du travail, Paris, Economica, coll. Approfondissement de la connaissance économique, 305 p.

Mucchielli J-L., 1998, Multinationalisation et mondialisation, Paris, Seuil, coll. Points, 373 p. 
Pecqueur B., 2005, « La « clusterisation » de l'économie mondiale », Sciences humaines, hors-série, p.48-55.

Porter M., 1993, L'avantage concurrentiel des nations, Paris, InterEditions, 883 p.

Ricardo D., 1815, Essai sur l'influence des bas prix du blé sur les profits du capital (An Essay on the influence of a law Price of Corn on the Profits of Stock showing the Inexpediency of Restrictions on Importation), Gallica, $17 \mathrm{p}$.

Scott A.J., 2001, Les régions et l'économie mondiale, Paris, l'Harmattan, Théorie sociale contemporaine, $187 \mathrm{p}$.

Subra P., 2007, Géopolitique de l'aménagement du territoire, Paris, A. Colin, coll. Perspectives Géopolitiques, 327 p.

Veltz P., 2002, « Les mutations du système productif. La géographie économique française aujourd'hui », Les Cahiers français, $\mathrm{n}^{\circ}$ 311, p.26-31.

\section{NOTES}

1. . Echaudemaison C.D., 1989, Dictionnaire d'économie et de sciences sociales, Paris, Nathan, p. 143.

2. . Arthuis J., 1993, Rapport d'information sur l'incidence économique et fiscale des délocalisations hors du territoire national, des activités industrielles et de service, Sénat, rapport n 337, $231 \mathrm{p}$.

3. . L'installation en Ecosse a fait l'objet d'âpres négociations puisqu'elle a été conditionnée par un gel de salaires pendant un an, une plus grande flexibilité du travail et des mesures d'incitation fiscale.

4. . L'usine était pourtant ouverte depuis 1930.

5. . Pour une analyse plus précise, on pourra se reporter à l'ouvrage intitulé : 40 ans d'aménagement du territoire. DATAR, La Documentation française, Paris, 2006, 158 p. On pourra également s'intéresser aux ouvrages de Philippe Subra sur la notion de territoire dans l'aménagement de l'espace. Subra P., 2007, Géopolitique de l'aménagement du territoire, Paris, A. Colin, coll. Perspectives Géopolitiques, $327 \mathrm{p}$.

6. . Pour une analyse plus détaillée, on pourra se reporter à l'ouvrage de Jacques Marseille. Marseille J., 2005, Empire colonial et capitalisme français, Paris, Albin Michel, collection Bibliothèque De L'évolution De L'humanité, 638 p.

7. Selon les termes de Jean-Claude Karpeles, Délégué Général de la fédération des industries électriques, électroniques et de communication.

8. . Pour une analyse plus précise, on pourra se reporter aux travaux de Milton Friedman (2005) qui s'intéressent particulièrement aux conséquences de la bulle Internet dans le cas de la soustraitance des services en Inde.

9. . Carroué L., 2002, Géographie de la mondialisation, Paris, colin coll. U, 364 p.

10. . Si l'investissement représente moins de $10 \%$ du capital de l'entreprise, on parlera plutôt d'investissement de portefeuille (IP). Pour une analyse plus précise on pourra se reporter à l'ouvrage d'Oliver Bouba-Olga Les nouvelles géographies du capitalisme. Comprendre et maîtriser les délocalisations. Paris, Seuil, 2006, 241 p.

11. . Ricardo D., 1815, Essai sur l'influence des bas prix du blé sur les profits du capital (An Essay on the influence of a law Price of Corn on the Profits of Stock showing the Inexpediency of Restrictions on Importation, Gallica, $17 \mathrm{p}$.

12. Sur la période, l'emploi net de ces entreprises a progressé de $4 \%$. Si l'on prend en compte leur activité en France, qui n'a progressé que de $3 \%$ et l'augmentation de la productivité qui a été en moyenne de $3.5 \%$, ces groupes auraient dû supprimer des emplois. 
13. Assemblée des chambres françaises de commerce et d'industrie, Délocalisations : la peur n'est pas une solution, Enquête auprès de 100 entreprises moyenne,. Novembre 2005, $79 \mathrm{p}$.

14. . Aubert P., Sillard P., 2005, Délocalisations et réductions d'effectifs dans l'industrie française, Document de travail de la DESE, Paris, Insee, $47 \mathrm{p}$.

15. Ils considèrent que les délocalisations sont détectées lorsque l'emploi diminue ou disparaît au sein d'un établissement et que les importations du groupe détenant l'établissement touché augmentent pour le type de biens auparavant produits en France.

16. . Fontagné L., Lorenzi J.H., 2005, Rapport désindustrialisation et délocalisation, Conseil d'analyse économique, $400 \mathrm{p}$.

17. . Fontagné L., D'isanto A., 2013, Chaînes d'activités mondiales: Des délocalisations d'abord vers l'Union européenne, INSEE Première, $\mathrm{n}^{\circ} 1451,4 \mathrm{p}$.

18. Direction du Trésor, 2012.

19. . L'Observateur de l'OCDE N² 292 T3 2012

20. . Demmou L., 2010, La désindustrialisation en France. Direction générale du trésor et de la politique économique (DGTPE), document de travail, numéro 2010/01, 49 p.

21. . Laine F., 2005, Secteurs et métiers industriels: l'industrie n'est plus ce qu'elle était, Premières synthèses et informations de la DARES, Ministère de l'Emploi, du Travail et de la Cohésion sociale, $\mathrm{n}^{\circ}$ 16.2.

22. . On pourra se reporter à l'article de Solange MONTAGNE VILETTE. Montagné Vilette S., 2001, « Le secondaire est-il soluble dans le tertiaire? »Annales de géographie, $n^{\circ}$ 617, p. 22-37.

23. . Selon la terminologie employée par C.A Michalet (1976). Le cabinet de consultants Capgemini appelle cette localisation optimale le « right shore ».

24. . Bost F., Delapierre M., 2007, «Formation des réseaux internationaux de production et reconfiguration des territoires ", Economies et sociétés, vol. 41, n², p.237-254.

25. . Bernie Vonderschmitt, PDG de l'entreprise américaine de semi-conducteur FPGA Xilinx est l'un des premiers à avoir appliqué le principe du fabless.

26. . Produit fini : résultat de la transformation d'une matière vers un produit prêt à être utilisé.

27. La Loi Barnier de 1993 impose au dernier occupant d'assumer les coûts de dépollution. Il reste pourtant le problème posé par les sites dits « orphelins » dont les derniers exploitants sont insaisissables ou insolvables.

28. . Porter M., 1993, L'avantage concurrentiel des nations, Paris, InterEditions, 883 p.

29. 800 millions d'euros ont été partagés entre toutes ces entités sur trois ans.

30. .www.aerospace-valley.com

\section{ABSTRACTS}

Competition across territories has shaken the French industrial landscape. The economic survival of some industrial companies was achieved at the expense of a spatial reorganisation commonly referred to as "relocation". These relocations first affected jobs where workforce constituted an important part of the cost of returns. Such activities went through difficult paths and led to numerous factory closures as well as thousands of job losses. Today, this scheme tends to evolve due to emerging countries' willingness to position on technical and technological activities. Because of the crisis environment in july 2008, it's interesting to think again about 
french offshoring. What's the link between offshoring and deindustrialisation? What are the repercussions on french territory?

L'ouverture et la mise en concurrence des territoires ont bouleversé le paysage industriel et économique français. La survie économique de nombreuses entreprises s'est faite au prix d'un redéploiement spatial de leurs activités appelé communément «délocalisation». Ainsi, les délocalisations ont d'abord affecté les métiers où la main-d'œuvre constituait une part importante du coût de revient. Ces activités ont connu des moments très difficiles et ont eu pour conséquences de nombreuses fermetures d'unités de production et la perte de dizaines de milliers d'emplois. En effet, certaines régions ont vu partir vers l'étranger nombre de leurs activités traditionnelles, tandis que d'autres filières sont en sérieux déclin (textile, habillement, cuir, jouet, électroménager, équipements automobiles par exemple). Aujourd'hui, ce schéma tend à se modifier par la volonté des pays émergents à se positionner aussi sur les activités techniques et technologiques. Le nouveau contexte international apparu en juillet 2008 à la faveur de la crise financière et économique justifie que l'on s'intéresse à nouveau à la question des délocalisations d'activités dans le cas français. Nous analyserons la participation des délocalisations aux mutations des systèmes productifs français. Quel est le poids des délocalisations dans la désindustrialisation du territoire? Quelles conséquences sur le tissu économique français et les territoires?

Die Öffnung und der Wettbewerb auf den Märkten hat die industrielle und wirtschaftliche Landschaft verändert. Der Preis des ökonomischen Überlebens zahlreicher Unternehmen war die räumliche (geografische) Umstrukturierung, bekannt als „Produktionsverlegung“. Betroffen sind folglich vor allem die Bereiche, in denen die Arbeitskraft einen großen Teil der Selbstkosten ausmacht. Diese erlebten schwierige Zeiten und als Konsequenz auch Schließungen von Produktionseinheiten und den Verlust zehntausender Arbeitsplätze. In der Tat mussten bestimmte Regionen mit ansehen, wie etliche ihrer traditionellen Produktionen ins Ausland wanderten oder sich ernstlich abwärts bewegen (zum Beispiel Textil-, Bekleidungs-, Leder-, Spielzeug-, Haushaltsgeräte- oder Autozubehörindustrien). Der neue internationale Kontext ergab sich im Juli 2008 durch die Finanz- und Wirtschaftskrise und löste in Frankreich ein erneutes Interesse an Verlagerungen aus. Wir müssen feststellen, dass die Verlagerung ein Teil des Wandels im französischen Produktionssystems geworden ist. Wie groß ist der Anteil der Produktionsverlagerung am Strukturwandel? Welche Konsequenzen sind für das wirtschaftliche Geflecht Frankreichs und der Regionen zu erwarten?

\section{INDEX}

Schlüsselwörter: Auswanderung der Firmen, Industriestadt, Politik gestutzt, Verfall der Industrie

Keywords: attractiveness, enterprises, globalization, industrial city, outsourcing, relocation Mots-clés: attractivité, délocalisation, désindustrialisation, entreprises, mondialisation, relocalisation, ville industrielle

\section{AUTHOR}

\section{DALILA MESSAOUDI}

Docteure en géographie, laboratoire CEMOTEV, UVSQ - 47 bd. Vauban 78047 Guyancourt dalilamessaoudi@hotmail.fr 